\title{
Procesos de innovación social (IS) como fuente de transformación social de comunidades rurales ${ }^{1}$
}

\author{
Cindy Arcos Soto ${ }^{2}$, Marlén Suárez Pineda ${ }^{3} \&$ Sandra Milena Zambrano Vargas ${ }^{4}$ \\ Universidad Pedagógica y Tecnológica de Colombia (UPTC), Colombia
}

Recibido, mayo 19 de 2015

Concepto evaluación, agosto 03 de 2015

Aceptado, octubre 15 de 2015
Referencia: Arcos Soto, C.; Suárez Pineda, M.; Zambrano Vargas, S. (2015). "Procesos de innovación social (IS) como fuente de transformación social de comunidades rurales". Revista Academia y Virtualidad, 8, (2), 85-99

\section{Resumen}

Este artículo tiene como propósito hacer una reflexión acerca de la importancia de la innovación social para el desarrollo social local, económico y cultural de las comunidades rurales. Para ello se desarrolla el concepto de innovación social estableciendo su relación con la transformación social favorable para dichas comunidades, desde perspectivas económicas y sociales; de otro lado, se muestran experiencias de innovación social exitosas en contextos rurales, destacando los logros alcanzados por dichas comunidades con relación a diversos aspectos como el desarrollo del liderazgo, la toma de decisiones en conjunto, el empoderamiento de los individuos, la reconstrucción del territorio y la construcción de visiones de futuro compartidas. Finalmente, se resaltan las dificultades existentes para el desarrollo de comunidades rurales que se constituyen en retos para la innovación social.

Palabras clave: innovación social, comunidades rurales, cambio organizacional, desarrollo local.

\section{Social innovation processes as a basis of social transformation of rural communities}

\begin{abstract}
This paper aims to reflect on relevance of social innovation for local, economic, and cultural development of rural communities. Firstly, we take the social innovation concept by establishing its relationship with a favorable social transformation to such communities from economic and social perspectives; secondly, experiences of successful

1. Artículo de reflexión como resultado del proyecto de investigación "Innovación social como base para el desarrollo empresarial, caso pequeños hatos lecheros en la provincia Centro en el departamento de Boyacá", del Grupo de Investigación IDEAS, en la línea de investigación Desarrollo Empresarial y agroindustrial.

2. Administradora de Empresas, UPTC; Integrante Grupo de Investigación IDEAS adscrito a la Escuela de Administración de Empresas de la UPTC Tunja. Correo: cindy.arcos@uptc.edu.co

3. Administradora de Empresas, UPTC; Especialista Negocios Internacionales; Magíster, Administración de Empresas; Docente, Escuela de Administración de Empresas; Directora Grupo de Investigación IDEAS. Correo: marlen.suarez@uptc.edu.co.

4. Ingeniera Industrial, UPTC; Especialista, Gerencia Talento Humano, UPTC; Magíster Administración, Universidad Nacional de Colombia; Docente, Escuela de Administración de Empresas; Integrante Grupo de Investigación IDEAS. Correo: sandra.zambrano01@uptc.edu.co
\end{abstract}


social innovation in rural environments are shown by highlighting achievements of these communities concerning several features, such as leadership development, making decisions together, empowering individuals, rebuilding countryside, and building shared future visions; finally, we highlight critical issues to develop rural communities that impose challenges against social innovation.

Keywords: social innovation, rural communities, organizational change, local development.

\section{Processos de inovação social como fonte de transformação social de comunidades rurais.}

\section{Resumo}

Este artigo tem o propósito de fazer reflexão ao redor da importância da inovação social para o desenvolvimento social local, econômico, cultural das comunidades rurais. Consequentemente se desenvolve o concepto de inovação social estabelecendo sua relação com a transformação social favorável para tais comunidades, desde perspectivas econômicas e sociais; de outro lado, mostram-se experiências de inovação social que fizeram sucesso (baseadas no social) em contextos rurais, destacando os logros atingidos por tais comunidades em relação aos diversos aspetos como o desenvolvimento de liderança, a tomada de decisões conjuntas, o ganho de poder dos indivíduos, a reconstrução do território e a construção de visões compartilhadas de futuro. Finalmente, ressaltam-se as dificuldades existentes para o desenvolvimento de comunidades rurais que constituem-se em retos para a inovação social.

Palavras chave: inovação social, comunidades rurais, mudança organizacional, desenvolvimento local.

\section{Introducción}

El mundo de hoy demanda soluciones efectivas que permitan el mejoramiento de las condiciones de vida (transformación social) de las comunidades en diferentes escalas: económica, social, laboral, familia y cultural. Por ello, los gobiernos, el sector productivo e incluso las mismas comunidades han tenido que ir construyendo estrategias para hacer frente a problemáticas de diversa índole que puedan dificultar su desarrollo.

Por esto han surgido procesos de innovación social (IS), que involucran a diversos actores, lo que les ha permitido a las comunidades ampliar sus capacidades (en asociaciones o agrupaciones), como es el caso de la habilidad de liderazgo local participativo. Este tipo de capacidades contribuyen a la construcción de capital social a partir de un reconocimiento de las realidades propias de la comunidad y las relaciones con su entorno, al punto que sus miembros se logran movilizar de manera conjunta en la toma de decisiones para promover iniciativas que faciliten resolver problemáticas locales (Azuero, 2009).

En la última década, diferentes países han empezado a adoptar modelos o prácticas de innovación social a la par de la innovación tecnológica, siendo estos conceptos diferentes entre sí.

En general, estos tipos de innovación se distinguen fundamentalmente por los fines que persiguen, pues el primero tiene que ver directamente con el cambio social y las relaciones entre las gentes, mientras que el segundo pretende acelerar los procesos productivos a la par del desarrollo y avance del transporte y los medios de comunicación disminuyendo las barreras territoriales, con el fin de crear nuevos productos y mejorar e incursionar en nuevos mercados (Martner, 1995); en sí, la innovación tecnológica se basa en la transformación de procesos 
Cindy Arcos Soto, Marlén Suárez Pineda \& Sandra Milena Zambrano Vargas

industriales y comerciales, creación de nuevos productos y servicios, incluso mejorar los ya existentes. No obstante, han existido dificultades para diferenciar los fines en estos dos tipos de innovación.

En este sentido, el concepto de innovación social se ha venido estudiando desde una perspectiva económica, en cuanto genera rendimientos tangibles en el tiempo, que ha dejado de lado su esencia fundamental: impactar las relaciones de los individuos, en la generación del cambio a partir del empoderamiento de las comunidades, en el trabajo colaborativo y en la manifestación de trabajo colectivo para la cohesión social en la búsqueda de visiones de futuro compartidas (Kliksberg, 1997).

La innovación social al encargarse de los individuos de una comunidad tiende a tener un carácter axiológico en cuanto a formas, costumbres y percepciones de vida de las personas, por ello este tipo de innovación se enfoca en valores sociales, como el bienestar, la calidad de vida, la inclusión social, la solidaridad, la participación ciudadana, la cooperación, la calidad medioambiental, la atención sanitaria, la eficiencia en la prestación de servicios públicos y el nivel educativo, los cuales contribuyen a transformar una sociedad, en la medida en que mejoren las condiciones de vida de sus integrantes; a la vez que aporta ventajas competitivas entre diferentes sociedades ante las diversas manifestaciones de la globalización.

El éxito de un proceso de innovación existe en primer lugar por la aceptación social de las iniciativas de innovación, por otra parte está en su utilización efectiva y continua, que se define como tal donde los usuarios de la comunidad emplean las innovaciones en su día a día, es decir su vida cotidiana. (Vera y Lavalle, 2010).

\section{Innovación social y transformación social, una descripción conceptual}

Durante el siglo XXI, alrededor del mundo se han venido consolidando el concepto y la práctica de la innovación en diferentes escenarios: científico, tecnológico, económico, político, cultural y social, los cuales han transformado gradualmente los procesos de desarrollo de los territorios, las industrias y las personas inmersas en una comunidad determinada.

En este sentido, aunque el concepto de innovación se ha asociado frecuentemente con procesos de creación $\mathrm{y}$ mejoramiento de productos (bienes y servicios); en realidad, éste tiene diversas aplicaciones relacionadas con la forma de hacer negocios, la generación de nuevos mercados y las modalidades de distribución. Estos mismos usos se presentan cuando se habla de innovación social aunque en este caso se presenta como fenómeno que pretende mejorar las condiciones de vida de comunidades en situaciones cambiantes (Fundación de la Innovación Bakinter, 2009).

Las primeras definiciones y aplicaciones acerca de la innovación surgieron con el fin de mejorar las condiciones tecnológicas y científicas de las organizaciones y los países, de tal forma que puedan responder ante las necesidades y desafíos que trae consigo la globalización. Este fenómeno ha impuesto ritmos acelerados para la resolución de problemas y hábitos de vida dinámicos y cada vez más sofisticados; esto se ve en la actualidad en cuestiones como la velocidad de las telecomunicaciones, las cuales no tienen fronteras y permiten el flujo de información entre diferentes grupos económicos, sociales y culturales (Asian y otros, 2013).

Dado lo anterior se puede entender qué tema de la innovación comienza en un escenario primordialmente económico (capitalista) y científico-tecnológico que persigue el desarrollo económico de las industrias y las naciones. Por ello, es claro que el propósito inicial de la innovación como proceso ha sido generar ventajas competitivas en un mundo cambiante (Córdoba y otros, 2014).

No obstante, autores como Jiménez y Morales (2012) afirman que los cambios en el diseño y fabricación de un bien o servicio deben enfocarse directamente en resolver o abordar problemas sociales y no simplemente en crear beneficios para sus propietarios, creando así una diferencia entre lo que se entiende como innovación 
social e innovación empresarial, siendo la segunda más de beneficio para los empresarios que para los consumidores. De esta manera, así como la innovación científica y tecnológica ha generado grandes aportes a la resolución de problemas del conocimiento, industriales y empresariales a través de productos tangibles, tales como patentes de productos o servicios, propiedad intelectual en la generación de nuevo conocimiento, desarrollo de nuevos procesos de calidad, empresas spin off, etc., se espera que la innovación social empiece a dar respuesta a los problemas de carácter social y comunitario y genere en las comunidades productos intangibles como el capital social (Posada, 2006; Durston, 2000). Esto se considera fundamental puesto que en este tipo de innovación están involucrados los procesos sociales entre los pueblos y las interacciones entre individuos.

Así, aunque el término de innovación social es relativamente novedoso, existen diferentes conceptos de esta expresión, desde posturas que se enfocan en la generación de nuevos productos o servicios hasta las que generan un impacto en la solución de problemas sociales. Algunas de estas definiciones se muestran en la tabla 1.
En suma, la innovación social, según Hernández y Sánchez (2014), se fundamenta en provocar cambios profundos en estructuras sociales complejas que permitan generar una mejor situación en uno o varios aspectos de relevancia para la vida de comunidades, que generalmente se encuentran en una posición contraria al disfrute efectivo de sus derechos.

Dentro de algunos de los beneficios intangibles que genera la IS se encuentran la solidez y unión de los integrantes de una comunidad, la construcción de lazos sociales, el fortalecimiento de la identidad regional, la iniciativa y desarrollo de proyectos comunes, la valoración de memoria histórica y cultural, la generación de equidad y participación y el desarrollo de relaciones cívicas sanas y pacíficas.

En otro sentido, la innovación social se distingue fundamentalmente de la innovación empresarial (científica-tecnológica) en cuanto a los indicadores de medición de sus resultados, puesto que este último tipo de innovación genera productos tangibles, que facilitan su medición a través de indicadores cuantitativos, mientras que la innovación social cuenta con beneficios

\begin{tabular}{|c|c|}
\hline Autor (Año) & \multicolumn{1}{|c|}{ Definición } \\
\hline Mumford (2002) & $\begin{array}{c}\text { Define la innovación en términos de colaboración donde hay una interacción de diferentes actores, } \\
\text { que parte de un objetivo o meta en común, en la cual nacen nuevas ideas acerca de las formas como } \\
\text { deben organizarse las personas para alcanzar los fines que persiguen. }\end{array}$ \\
\hline Nilson (2003) & $\begin{array}{l}\text { Define innovación como generador de un cambio sistémico, es decir la manera como una } \\
\text { sociedad intenta encontrar vías o alternativas para solucionar problemas profundos, difíciles y } \\
\text { complejos como la pobreza, la salud, la calidad del medioambiente, la educación, la violencia. }\end{array}$ \\
\hline Bacon y otros (2008) & $\begin{array}{l}\text { Se trata de nuevas ideas (productos, servicios y modelos) desarrolladas para cumplir necesidades } \\
\text { básicas no satisfechas. }\end{array}$ \\
\hline Phills y otros (2008) & $\begin{array}{l}\text { Novedosa solución a un problema social que es más efectiva, eficaz, sostenible o justa que las } \\
\text { soluciones existentes, y por la cual el valor creado se transfiere a la sociedad en su conjunto y no } \\
\text { a manos privadas. }\end{array}$ \\
\hline Westley (2009) & $\begin{array}{l}\text { Iniciativa, producto, proceso o programa que cambia profundamente las rutinas básicas, los } \\
\text { recursos, la autoridad o las creencias de cualquier sistema social. }\end{array}$ \\
\hline Murray y otros (2010a) & $\begin{array}{c}\text { Define la innovación como un bien común, en términos de valores compartidos, donde mejoran la } \\
\text { capacidad de la sociedad para actuar, donde surgen nuevos productos, servicios o procesos capaces } \\
\text { de atender necesidades sociales, creando nuevas colaboraciones. }\end{array}$ \\
\hline
\end{tabular}

Tabla 1. Definiciones de innovación social

Fuente: elaboración propia a partir de Buckland y Murillo (2013 y 2014). 
intangibles. Estos últimos tienden a ser de carácter cualitativo y son complejos de visibilizar en cuanto a sus resultados (impactos sociales generados) pues se tratan de activos intangibles que se manifiestan en los territorios, como consecuencia de las relaciones sociales entre sus miembros (Caravaca y otros, 2003; Caravaca y otros, 2005).

Por esta razón, en la actualidad se carece de modelos y metodologías estándar, con un enfoque cualitativo, que permitan evaluar el impacto de la innovación social en los procesos sociales de las comunidades intervenidas. En este sentido, aunque varios autores han considerado algunas variables para evaluar tanto las contribuciones de la innovación social y su potencial como la relación que ésta tiene en relación con la apropiación y generación de conocimiento (Reyes, 2011), existe muy poco consenso en torno a cómo medir la innovación social y la forma de definir el impacto (transformación) social de ésta. También hay una sorprendente falta de uniformidad en los indicadores y metodologías de los informes (Buckland y Murillo, 2013). Todo ello se da a pesar de la riqueza y la vitalidad del sector.

Pese a lo anterior existen diferentes métodos para medir el impacto de la innovación social pues en diferentes países han emergido algunos parámetros para avalar e implementar procesos de IS que consideran sus realidades actuales y, con ello, se pretende que dichos procesos respondan realmente a los requerimientos de sus habitantes y contribuyan a resolver y atender sus necesidades insatisfechas. Uno de estos métodos se referencia en la parte relacionada con las experiencias exitosas.

Por otro lado, Murray y otros (2010b) - citado en Buckland y Murillo (2013) - describe cuatro actores económicos principales que, en su interacción, determinan los resultados y consecuencias de las innovaciones sociales en la humanidad:

[...] El mercado cada vez está más comprometido con la economía social por medio de programas de RSE o de áreas específicas como el comercio justo y los bienes medioambientales, la familia también alberga ciertas ambiciones personales, pero participa en la economía social a través de redes, asociaciones y movimientos sociales informales, las posiciones binarias entre mercado y estado se están contextualizando en un conjunto más complejo de relaciones a medida que el mercado se adentra en el estado, el estado en el mercado, y ambos establecen nuevas relaciones con la sociedad civil y la economía del subsidio.

La innovación social surge de iniciativas propias de los mismos individuos, desde las comunidades, que desean de manera voluntaria aportar soluciones novedosas o renovadas para mejorar su nivel de vida y para desarrollar iniciativas sociales. Estos procesos de innovación social requieren una estructura organizacional que los soporte en cuanto al direccionamiento y gestión de recursos humanos, financieros y administrativos de tal manera que permitan a las innovaciones no sólo multiplicar sus beneficios a las comunidades directamente implicados sino también a otras organizaciones (asociaciones, cooperativas, redes empresariales, ONG).

Por otra parte, este tipo de organizaciones deberían tener en cuenta algunas dimensiones que permitan dar respuesta a aquellas necesidades que merecen ser atendidas en las comunidades; Orrego y Arboleda (2006) proponen dos dimensiones: una social ética y una cultural.

Para que una innovación social se materialice en resultados que generen impacto social existen procesos que intervienen o facilitan, que ellos se den de manera directa e indirecta. A continuación se nombran cuatro de ellos junto con las categorías que los conforman (Gutiérrez, 2009).

- Procesos mentales, los cuales incluyen un crecimiento y pensamiento divergente, visión global o perspectiva sistémica.

- Procesos actitudinales, los cuales implican habilidades como apertura y tolerancia, valentía y riesgo, empatía.

- Procesos sociales, que involucran diferentes actores, como diversos saberes, el cual conduce a una mul- 
tidisciplinariedad y diversidad, además promulga la integración.

- Procesos instrumentales, hace posible el isomorfismo empresarial, permite el trabajo en red, rescata la importancia del territorio, el acercamiento de nuevas tecnologías en general y la web 2.0 en particular.

Por otra parte, dado que la innovación social se entiende como un proceso que involucra la relación de las gentes que trabajan de manera cohesionada para resolver problemas propios de la comunidad, se hace preciso hablar aquí sobre las dinámicas organizacionales que soportan dichos vínculos

La transformación social tiene que ver con un cambio exigido por las personas o la sociedad, es un cambio de la gente para la gente, un cambio que se define así mismo en un contexto de injusticia, de desigualdades, de falta de recursos y acceso a los mismos.

La transformación social implica un cambio desde el interior de procesos, instituciones y fundamentalmente de las personas, materializándose en acciones concretas; a la vez requiere compromiso de los actores involucrados, el cual desemboca en un desarrollo, no sólo en términos de crecimiento económico, convivencia y paz, reparto de la riqueza y el conocimiento, también implica la capacidades humanas en lo relacionado con las condiciones sociales, la cultura, las costumbres incluso lo que hay en el interior de las personas, sus imaginarios y expectativas de vida, buscando siempre el desarrollo humano en todas las dimensiones, por ejemplo las igualdad de oportunidades, políticas justas y coherentes, empleabilidad, entre otras. Así lo señala el pacto por una economía responsable y sostenible (s.f.)

De lo anterior hay grupos y asociaciones que no sólo se limitan a denunciar la desigualdad, la explotación, el injusto reparto de la riqueza en el mundo, aunque también es importante señalar que por derecho no pueden renunciar a esto, a pesar de ello son personas que tienen o desarrollan una capacidad propositiva, defendiendo y creando formas ,métodos y valores para enfrentar diversas situaciones, donde en comunidad se buscan alternativas de producción y consumo, educación, condiciones y cuidados de la salud, convivencia ciudadana, que creen una mejor sociedad posible ya que debe ser la misma comunidad la que desarrolle sus propias capacidades de su poder social, quien produzca, consiga, recupere, recicle y reutilice los medios necesarios para hacer partícipe de una manera efectiva de sus miembros.

Allí en la comunidad se hace necesario multiplicar las posibilidades del encuentro en el conocimiento mutuo, el intercambio de experiencias entre la mismos miembros de la comunidad y comunidades externas, que vayan encaminadas a relaciones de confianza para la continuidad y sustento de la sociedad que geste iniciativas de innovación social, de lo que se trata es de trabajar en conjunto, formarse en unión, aprender a compartir conocimientos, objetivos, recursos, proyectos, logros y fracasos.

Hoy en día existen colectivos que apuestan a una sociedad diferente, por ello han nacido diversas formas de aprendizaje, innovaciones, alternativas creativas, de aprendizaje y participación, la cooperación y la inteligencia colectiva que busca responder a los desafíos y retos de un mundo cambiante y en crisis. (De la Riva, 2012).

De acuerdo con la UNESCO (2015), las comunidades rurales son grupos de personas que habitan en regiones donde su actividad principal es la agricultura; en su condición de campesinos, pastores, nómadas o pescadores se ocupan de la cría de animales, la transformación y comercialización de alimentos y otros productos y servicios derivados del agro.

Los pobladores rurales representan el 70\% de la población mundial y el $72 \%$ de los habitantes de los países menos desarrollados. Las desigualdades entre el campo y la ciudad constituyen un obstáculo importante para el desarrollo sostenible. Las zonas rurales comprenden asentamientos humanos de menos de 10.000 habitantes y en el espacio rural predominan las granjas, los bosques, los ríos y lagos, las montañas o el desierto. 
Cindy Arcos Soto, Marlén Suárez Pineda \& Sandra Milena Zambrano Vargas

Las comunidades rurales para llevar a cabo procesos de innovación social están dadas a relacionarse con diferentes actores en diversos contextos, así: en el núcleo familiar, donde se dan relaciones de intimidad, de conyugalidad y parentalidad. En un vecindario se reflejan relaciones personales y de sociabilidad. Con empresas se brindan relaciones de trabajo y de producción. Con instituciones de dan relaciones de organización y ciudadanía.

En el caso de los países latinoamericanos, las comunidades rurales campesinas están constituidas por el núcleo familiar cercano donde se dan relaciones de intimidad, personales y de trabajo, y ante los diversos cambios de la sociedad experimentan algunos de los cambios sociales que han tenido se encuentran la concepción de familia y las relaciones de género.

Synger (2012) plantea que los esquemas tradicionales $\mathrm{y}$ estables en relación con el ámbito doméstico y profesional, con base en relaciones patriarcales, ha ido cambiando, dando paso a nuevas formas de convivencia con altos índices de monoparentalidad y la nueva relación empleo-familia donde los roles tanto del hombre como de la mujer han ido cambiando en la actualidad, donde en algunos países se han ido formulando políticas para prestar servicios a las familias, educación infantil, residencias para personas de la tercera edad, programas de empleo equitativo para ambos géneros, lo cual impacta en los programas de bienestar de los gobiernos actuales.

\section{Algunas experiencias de éxito en innovación social}

Los casos de éxito son testimonios vivos de los beneficios (transformaciones sociales) que traen a las comunidades el desarrollo de iniciativas de innovación social; sin ellos se carecería de credibilidad, replicabilidad y desarrollo de este tipo de perspectiva. Por ello, en esta parte se muestran algunas experiencias de éxito que revelan las bondades de la innovación social para lograr la transformación favorable de las comunidades (en particular, las rurales). Todo esto es de gran utilidad en función de poder conocer, analizar y reproducir aquellos conocimientos adquiridos por las comunidades y de rescatar los logros obtenidos en estos casos, bien sea para aplicarlos en otro tipo de comunidades o como medio de incentivar a todos aquellos que puedan tener una iniciativa en mente para su desarrollo e implementación.

Al respecto existen diferentes estudios sobre innovación social en comunidades rurales que ya han presentado algunos de los beneficios y logros que estos procesos generan para los participantes y para la transformación social de las comunidades. A continuación se mencionan sólo algunos de ellos.

En Colombia, la Anspe -Agencia para Superación de la Pobreza Extrema- junto con la colaboración del DPS -Departamento de la Prosperidad Social-y otras instituciones en el país ha recopilado dos catálogos "Hilando", los cuales muestran prácticas y experiencias de innovación social", entre la cuales se pueden destacar las siguientes (catálogo Hilando 2012 \& 2013):

- Eco techos productivos, el proyecto busca mejorar condiciones de habitabilidad y seguridad alimentaria, promueve escenarios de educación ambiental, y fortalece espacios comunitarios y familiares con la implementación de techos verdes en viviendas de interés prioritario, en la zona de Soacha Cundinamarca. Es un caso de innovación social porque satisface necesidades de un grupo de población en una franja de alta vulnerabilidad, mejorando las condiciones de habitabilidad preservando el medio ambiente. Tiene un impacto también económico a través de la comercialización de hortalizas las cuales son cultivadas por las familias lo que les admite reducir los gastos de alimentación, a la vez que les permite una alimentación balanceada. Los logros alcanzados por esta iniciativa son: vincular a los beneficiarios de proyectos en procesos de formación, que les ha permitido generar recursos económicos para suplir algunas necesidades básicas de alimentación y vivienda, utilización de elementos de fabricación e insumos amigables con el medio ambiente.

- Innovación rural participativa, una estrategia para los pequeños agricultores, busca mejorar la calidad de vida de productores rurales a través de procesos participativos en la Región caribe, Andina y Orino- 
quia. Es innovación social porque busca atender a pequeños productores en situación de pobreza extrema a través de la implementación de una estrategia que les permita a ellos mismos solucionar en diferentes ámbitos lo organizativo, productivo y social, logrando empoderar a los agricultores a potenciar su autonomía para gestionar, planificar y ejecutar recursos. Los logros obtenidos de la iniciativa son: consolidación de saberes entre expertos y pequeños agricultores gestándose valores como la confianza, incremento de ingresos de la comunidad beneficiada con la iniciativa, como consecuencia de ello se han gestado mejores relaciones familiares y sociales, con el intercambio de experiencias nombradas anteriormente, los productores líderes hacen un acompañamiento a otras comunidades con necesidades similares, se han formado redes de trabajo a partir del uso de herramientas de información de tecnología y comunicación.

- Modelo de negocio para cruderos, un esquema alternativo en la comercialización de leche cruda, este modelo beneficia a población rural y urbana de Bogotá dedicada a la comercialización de leche cruda para consumo humano directo, dirigido a estratos 1, 2 y 3 . El modelo trabaja tres ejes principales, el primero es definir un plan de trabajo que reúna aspectos técnicos, financieros y administrativos que fortalezca la pequeña comercialización de leche, segundo beneficiar los actores involucrados en la comercialización y tercero fomentar actividades que incentivan y fortalezcan la cadena de producción y distribución de leche cruda. Es innovación social porque responde ante la necesidad de mejorar los procesos de producción y comercialización de leche cruda, garantizando un precio justo en un producto de calidad, este modelo empodera a la comunidad, ya que promueve la participación activa de los productores en cada una de las fases del proceso que se ha nombrado con antelación, especialmente el fortalecimiento organizacional y el desarrollo empresarial, al asistir a las jornadas de capacitación y al buscar nuevas alternativas de negocio propuestas por los mismos productores derivadas de la misma actividad lechera. Los logros destacados del modelo son: desarrollo exitoso de operaciones piloto, han realizado análisis de peligros y puntos críticos de control a partir de simulación de dinámicas de abastecimiento, distribución y comercialización de la leche. Diseño montaje y dotación de planta de enfriamiento de leche cruda. Impacto positivo de los ingresos de los productores con un alza del 23,2\% el precio del litro de leche. Mejoramiento de la calidad microbiológica de la leche. Buenas prácticas de ordeño dirigidos a orientar a los productores a diligenciar registros pecuarios.

- Modelo agro solidario, desarrollo socioeconómico para el fortalecimiento de la producción agroecológica de quinua, el modelo tiene la finalidad de mejorar la calidad de vida de las familias asociadas a la producción de quinua de AgroSolidaria Federación Boyacá, en total son 181 familias, el modelo busca mejorar los hábitos alimenticios de las estirpes cultivadoras, en cuanto a nutrición y salud, mejorando también el nivel de ingresos y las fuentes de trabajo. Este modelo asociativo permite promover la gestión productiva y empresarial mediante formación, capacitación y acompañamiento técnico en lo relacionado con la producción de la quinua. Es innovación social porque cuenta con procesos novedosos de círculos económicos solidarios (productores, transformadores, distribuidores y consumidores), la participación de la comunidad se basa en una estructura organizativa sólida, y en una gestión administrativa democrática, además tienen un sistema financiero autónomo, llamado "finanzas solidarias", asimismo tiene un enfoque de producción novedoso ya que posiciona la producción agroecológica de la quinua como un cultivo estratégico por ser tanto un alimento de alto valor nutricional, así como ser un elemento de identidad cultural del territorio. Dentro de los logros que se pueden resaltar se encuentran: implementación de un modelo de organización asociativo con base social, donde se concretan reuniones entre productores, se hace planeación de gastos y seguimiento de la producción conformado por comités y círculos solidarios, rescate del cultivo 
ancestral de la quinua al conmemorar una tradición cultural y gastronómica de la región incursionando en cultivos limpios. Aumento del número de familias asociadas, generación de nuevo conocimiento a través de ejercicios de socialización e intercambio de experiencias entre seccionales de la federación, apropiación de prácticas de participación solidaria que ha generado cohesión social mediante el trabajo en equipo entre productores y participación social a través de los círculos solidarios donde el productor se involucra en cada una de las fases del modelo.

- $\quad$ Pérez y Clavijo (2012), por su parte, muestran el caso de la corporación PBA (Desarrollo Participativo y Sostenible de los pequeños productores rurales), gracias a la indagación que promueve la FAO sobre sistemas de innovación agrícola en el mundo y en el caso concreto de la producción tradicional del ñame en la Región Caribe. En el estudio se muestra la forma cómo se gestó un fortalecimiento organizacional y empoderamiento en las comunidades gracias a los procesos inherentes a la innovación social. Estos procesos generaron beneficios en ejes principales como son: la infraestructura para su operación (con personal local a cargo de la misma, capacitado por las entidades miembros del consorcio en temas administrativos y de gestión); la motivación por logro (en la cual los productores afianzaron su autoestima mientras desarrollaron una conciencia colectiva que va más allá de las necesidades prácticas de individuos y organizaciones, más que recibir capacitación); el desarrollo organizacional y emprendimientos productivos (en donde se han logrado desarrollos organizacionales importantes, caracterizados por la creación y consolidación de Grupos Participativos Locales (GPL), que luego han trascendido a asociaciones que se han fortalecido en torno a los proyectos del Programa); el uso de las nuevas tecnologías de la información y comunicación (mediante acciones de empoderamiento han generado las capacidades y habilidades necesarias para enfrentar nuevos retos entre ellos el uso de las TIC, como alternativa de capacitación y comunicación entre los pequeños productores); y finalmente la creación de nodos (con proyectos que han impulsado la creación importante de redes entre agricultores e instituciones).

En Colombia, el Gobierno Nacional, junto con la Agencia Nacional de la Pobreza extrema ANSPE, el Observatorio Colombiano de Ciencia y Tecnología, Colciencias, y el DNP, encuentran el caso referido en un estudio titulado "Barreras e incentivos de la innovación social". En este caso, se buscó reflexionar sobre el tema y sensibilizar a los diferentes actores que persiguen y quieren desarrollar procesos de innovación social en Colombia que puedan aportar a la construcción y consolidación de una política pública en el país. En este sentido se realizó un taller sobre los retos de innovación social en Colombia; cinco proyectos fueron invitados a exponer sus experiencias innovadoras, identificando las principales barreras que han tenido durante el proceso con base en las percepciones de las personas involucradas en el proyecto y que participaron en mesas de trabajo durante el taller (Observatorio Colombiano de Ciencia y Tecnología, 2013).

\section{Otras regiones:}

- En Cataluña se muestra el ejemplo de la agricultura social, cuyo objeto es la creación de soluciones de empleo en agricultura para personas en riesgo de exclusión. En este caso el ideal, que fue apoyado por el gobierno, buscó incorporar personas con discapacidades o comunidades en riesgo para que en conjunto trabajen con proyectos productivos, ofreciendo alianzas sociales. Los resultados sin duda alguna han sido alentadores puesto que se han creado soluciones laborales en el marco de una economía más justa, ética y solidaria y mediante una economía al servicio de las personas, en donde prevalece la justicia del derecho a un empleo digno y con conciencia ecológica (González y otros, 2014).

- Otro ejemplo se presenta en el Municipio de Balacán (Tabasco, México) sobre innovación y capacidad de organización de cooperativas pesqueras, da cuenta sobre la capacidad organizativa de tres cooperativas, 
donde se muestra el nivel de tecnificación y de organización logrado en ellas durante el proceso de innovación. En este caso, la experiencia innovadora no se basa en una situación trasformadora en sí misma, sino que muestra cómo, a través de la capacidad organizativa, se logró contribuir en la creación de espacios de interacción donde pueden irse gestando las innovaciones (Cano y otros, 2012).

\section{Algunos avances en los factores para medir el impacto de la innovación social}

Gracias a los buenos resultados en experiencias como las señaladas previamente, en la última década han aparecido institutos, instituciones, centros de investigación, grupos de investigación e incluso algunos gobiernos que se han interesado en el tema y han adoptado políticas para generar desarrollo y conocimientos en torno a la innovación social aunque, como se mencionó previamente, ello no ha permitido aún un consenso o un modelo unificado que permita evaluar el impacto de las innovaciones a nivel local y territorial.

Sin embargo, como se refiere en Arévalo (2013), ya se cuenta con algunos factores identificados como relevantes para la evaluación de los beneficios de un proyecto de innovación social sobre las comunidades. Este autor muestra ciertos avances al respecto en algunas regiones, como América latina y el Caribe, a través de la CEPAL, que pueden ser considerados gracias a un estudio realizado en colaboración durante 5 años con la fundación W.K Kellog.

En este estudio, se señala el análisis que se hizo de 4.800 experiencias de innovación social en la región (sometidas a concurso de acuerdo con parámetros que los aliados construyeron a partir de las realidades concretas de la región por medio de un comité de notables, expertos en materia política y social con conocimiento en las problemáticas de dichas comunidades) con el fin de escoger aquellas iniciativas que lograron impacto social. Para esta evaluación se tuvieron como referencia ocho factores o parámetros a fin de determinar los proyectos ganadores (Tabla 2):

\section{Limitaciones a la transformación social de las comunidades rurales: retos para la innovación social}

Los procesos de innovación social, al involucrar recursos humanos, financieros, administrativos y tecnológicos, implican la necesidad de desarrollar una combinación de capacidades y habilidades que permitan que iniciativas o proyectos sean sostenibles en el tiempo y generen transformaciones sociales favorables para las comunidades intervenidas mientras generan modelos que puedan llegar a implementarse en otras que presenten problemáticas similares, sobre todo en cuanto se refiere a poblaciones rurales.

Para contribuir a este propósito, Martín (2000) hace alusión a algunos aspectos o variables claves, referidas al desarrollo (transformación) local en espacios rurales, que deben ser considerados en los procesos de innovación social, a saber: la identidad territorial, la imagen del campo y la ciudad, el acceso de mercados, la tecnología, las actividades de empleo, la gestión del espacio y de recursos naturales y el desplazamiento de habitantes rurales a la ciudad.

Teniendo en cuenta los aspectos claves para la transformación social en comunidades rurales, se hace preciso analizar cada uno de ellos para comprender la manera como éstos pueden convertirse en limitaciones al desarrollo de dichas comunidades a partir de los aspectos que señala Martin (2000):

- Movilización de población y cohesión social: este aspecto permite que los individuos generen espacios de reflexión colectiva sobre el desarrollo local.

- La limitante, respecto a este asunto se encuentra en la baja capacidad para congregar a las personas dado que, por diversas razones, muchos de ellos sólo quieren resultados inmediatos que les permitan solucionar diferentes problemáticas y otros sólo se sienten motivados a ir a reuniones si perciben la oportunidad de acceder a recursos económicos que se ajusten a su realidad agropecuaria o donde puedan 
acceder a alguna ventaja de carácter económico que les refleje mejora en el precio de sus productos.

- Identidad del territorio y la especificidad rural: esta variable se refiere a que las nuevas generaciones, es decir los hijos de los campesinos y productores en gran escala, mantengan las tradiciones. Este aspecto es una dificultad pues existe el riesgo de que estas nuevas generaciones pierdan las tradiciones o no mantengan continuidad con aquellas que caracterizan la vida del campo en cuanto a saberes ancestrales o modos de vida. Incluso existe el riesgo de que la identidad territorial se extinga como consecuencia de visualizar al campo como una forma de vida anticuada

\begin{tabular}{|c|c|}
\hline Factor & Descripción \\
\hline Innovación & $\begin{array}{l}\text { Hace referencia a que las iniciativas deben ser completamente novedosas: experiencias con } \\
\text { modificaciones significativas, planteamientos o procesos ya conocidos en otros contextos, cuando } \\
\text { tales cambios tuvieron gran impacto en los resultados obtenidos e implican un ajuste pertinente a } \\
\text { los planteamientos o procesos utilizados en proyectos similares. }\end{array}$ \\
\hline $\begin{array}{l}\text { Relación entre costo } \\
\text { y resultado }\end{array}$ & $\begin{array}{l}\text { Refiere a los costos requeridos para iniciar el proyecto y ejecutarlo, así como los resultados } \\
\text { obtenidos del mismo, con el fin de que los proyectos tengan una sostenibilidad en su ámbito } \\
\text { geográfico y en otros contextos, para así generar alianzas con entidades ya sean públicas o } \\
\text { privadas. }\end{array}$ \\
\hline $\begin{array}{l}\text { Potencial de } \\
\text { replicabilidad }\end{array}$ & $\begin{array}{l}\text { Está dirigido a detectar las formas eficientes y exitosas de enfrentar problemas persistentes en la } \\
\text { región de América Latina y del Caribe. De esta manera no sólo se resuelven problemáticas de las } \\
\text { comunidades directamente beneficiadas, sino territorios aledaños que presenten problemáticas } \\
\text { similares. }\end{array}$ \\
\hline $\begin{array}{l}\text { Potencial de } \\
\text { transformarse en } \\
\text { política pública }\end{array}$ & $\begin{array}{l}\text { Busca que una vez detectados los diversos e innumerables problemas que afectan a muchas } \\
\text { comunidades, a los cuales desafortunadamente no se le han dado soluciones institucionales, los } \\
\text { emprendimientos probados a pequeña escala puedan ser expandidos a varios territorios dentro de } \\
\text { la misma región con el fin de articular esfuerzos con el Estado para volverlo política pública, una } \\
\text { vez el proyecto sea declarado como factible y sostenible en el largo plazo. }\end{array}$ \\
\hline $\begin{array}{l}\text { Efectos sobre } \\
\text { determinantes de la } \\
\text { pobreza }\end{array}$ & $\begin{array}{l}\text { Permite evidenciar que no sólo la pobreza se genera por escasez de ingresos, sino también } \\
\text { por carencia de otros factores como oportunidades o discriminaciones. La disminución de los } \\
\text { indicadores de pobreza es clave ya que permiten cumplir con los objetivos del milenio de las } \\
\text { Naciones Unidas. }\end{array}$ \\
\hline $\begin{array}{l}\text { Potencial para reducir } \\
\text { la discriminación y la } \\
\text { exclusión }\end{array}$ & $\begin{array}{l}\text { Radica en establecer si el proyecto brinda soluciones que mejoren la calidad de vida de poblaciones } \\
\text { vulnerables en aspectos relacionados con el género, la edad, las características étnicas, la } \\
\text { pertenencia a estratos bajos, incapacidades físicas o mentales o el aislamiento geográfico. }\end{array}$ \\
\hline $\begin{array}{l}\text { Desarrollo de la } \\
\text { responsabilidad } \\
\text { social }\end{array}$ & $\begin{array}{l}\text { Pretende mirar si la innovación social suscita la participación del sector privado y empresarial } \\
\text { en la búsqueda de soluciones a problemas que afectan a los grupos menos favorecidos, donde } \\
\text { se quieren implementar prácticas de responsabilidad social corporativa. Se quiere que las } \\
\text { motivaciones y estrategias evidencien realmente el interés de las empresas por ayudar a grupos } \\
\text { vulnerables y no como una manera de extender los negocios de la empresa. }\end{array}$ \\
\hline $\begin{array}{l}\text { Potencial de } \\
\text { sostenibilidad }\end{array}$ & $\begin{array}{l}\text { Evalúa qué tanto es sostenible la iniciativa a largo plazo, es necesario que haya pasado por pruebas } \\
\text { de experimentación previas y superado dificultades de la puesta en marcha que permitan tener } \\
\text { resultados cuantitativos. } \\
\text { Implica continuar convocando a la comunidad de beneficiarios donde se plasme que continuarán } \\
\text { participando, contar con financiación por un periodo de tiempo razonable o contar con modelos } \\
\text { auto sostenibles y estar articuladas con otros actores, con la sociedad civil, con una comunidad, } \\
\text { con algún ente del gobierno local o nacional. }\end{array}$ \\
\hline
\end{tabular}

Tabla 2. Factores que permiten evaluar el impacto de la innovación social Fuente: elaboración propia a partir de Rey y Tancredi (2010). 
y atrasada, lo cual implica que las formas de vida campesina desaparezcan con el tiempo.

- Acceso a los mercados y la competitividad: este tema es crucial porque implica que las prácticas ancestrales de producción de la agricultura pueden verse afectadas debido a la apertura de nuevos mercados que hace que los clientes tengan mayores exigencias en precios, calidad y servicio (a la hora de adquirir productos y servicios). También puede ser una dificultad este aspecto ya que algunos productores se rehúsan a cambiar las prácticas tradicionales de su actividad agropecuaria ya sea porque tienen costumbres arraigadas o porque piensan que sale más costoso implementar mejores destrezas (como es el caso de las buenas prácticas agrícolas que exigen ciertos estándares de calidad).

- Acceso a la tecnología: en el contexto rural existe todavía una brecha en cuanto al acceso a herramientas informáticas y de telecomunicación, especialmente en los países subdesarrollados. Esto se puede dar al menos por dos razones: porque los índices de escolaridad de la población rural adulta es muy baja debido a las condiciones precarias de vida y porque los jóvenes que están recibiendo nuevos conocimientos en las escuelas en su mayoría quieren emigrar a las grandes ciudades como consecuencia de una visión del campo poco prometedora para su realización personal y profesional.

No cabe duda de que existen limitaciones a la transformación social favorable de las comunidades rurales, sobre todo en lo que tiene que ver con aquellas percepciones y formas de actuación de productores y campesinos que terminan configurando su manera de actuar en un contexto determinado. Uno de estos aspectos, tal vez el más frecuente y difícil de atacar, tiene que ver con la apertura hacia el aprendizaje pues este requiere de principios y valores que implican un alto nivel de compromiso (Chiriboga, 2003).

Todas estas dificultades plantean nuevos retos para la innovación social en función de lograr la transformación social de las comunidades, especialmente las rurales.
Entonces, queda un arduo camino por recorrer en función de valorar los diferentes tipos de conocimiento y formas de aprendizaje y de desarrollar un ambiente proclive que estimule la participación de los miembros de las comunidades en el proceso. Por otro lado, se requiere promover el diálogo y el encuentro de perspectivas y experiencias diferentes para generar procesos creativos.

\section{Reflexiones finales}

La innovación social es un cuerpo teórico en crecimiento; sin embargo, su valor se refuerza con las experiencias que a diario se observan en las comunidades; se necesita del empeño del Estado y del apoyo decidido de las universidades para continuar con estos progresos y poder dar soluciones a las problemáticas que nos aquejan y que sólo encuentran sentido si surgen desde el seno mismo de lo social, es así como se considera importante el fortalecimiento de la triada, en donde los grupos de investigación pueden aportar a las problemáticas sociales formulando proyectos en los que puedan participar de forma activa y asertiva.

De acuerdo con los avances en conceptos y teorías de innovación social se puede decir que ésta ha surgido como un proceso que tiende a resolver problemas de base social; es decir atiende necesidades insatisfechas como la pobreza, el mejoramiento de las relaciones sociales entre las gentes, la generación e inclusión laboral, por nombrar algunas de ellas, las cuales no han sido resueltas de manera mediática por los gobiernos $\mathrm{u}$ organismos competentes. Por tanto, la IS surge como fenómeno donde las comunidades se empoderan de tal forma que son las mismas gestoras y protagonistas de la transformación social de sus realidades.

Teniendo en cuenta las experiencias exitosas, fruto de procesos de base social, se visualiza claramente beneficios directos en las comunidades involucradas. Dentro de ellas se encuentran tanto incentivos económicos para la puesta en marcha de las iniciativas, la transformación en las relaciones sociales entre los miembros de las comunidades, donde se evidencia un cambio personal de los actores directamente involucrados, lo que significa que hay transformación cultural, allí se reflejan ciertas 
características, como una apertura mental, coherencia, creación y desarrollo de planes que conducen a objetivos claros para el desarrollo de proyectos conjuntos, a partir de la generación de ideas en grupo, lo que conduce a robustecer las relaciones entre los miembros de una colectividad, lo cual tiene como consecuencia que se disgreguen las barreras que causan envidia y el individualismo, la superación y temor al fracaso, así como la construcción de visiones de futuro compartidas durante el proceso.

A pesar de que no existen modelos estándar para evaluar los impactos de las innovaciones sociales se han hecho algunos acercamientos que ya determinan algunos factores a considerar en procesos de innovación social en función de lograr transformaciones benéficas en las comunidades. Se puede evidenciar entonces que existen beneficios reales e intangibles en las comunidades rurales en las que la IS se ha dado. Esta ha proporcionado la posibilidad de mejorar las condiciones de supervivencia y el entorno productivo de las comunidades.

En el caso latinoamericano habría que averiguar qué métodos se ajustarían mejor a la realidad de la región y que permitan evaluar el impacto real de la innovación social, de acuerdo con las realidades de cada uno de los países que la conforman, no sólo en términos cuantitativos, sino cualitativos. En este método se debería considerar el hacer énfasis en un enfoque más humanista que capitalista, para sentar un precedente que conduzca a los gobiernos $\mathrm{u}$ organismos bilaterales a plantear una política transversal de apoyo a iniciativas de base social, sobre todo en sectores vulnerables como la agricultura, la cual se ha visto transgredida por los efectos de la globalización.

Los procesos de innovación social le han permitido a las comunidades ampliar sus capacidades, como en el caso de la habilidad de liderazgo desde lo local y participativo, permitiendo la construcción de capital social, al punto de que sus miembros se logran movilizar de manera conjunta en la toma de decisiones, el empoderamiento de los individuos, la reconstrucción del territorio y la construcción de visiones de futuro compartidas para promover iniciativas que faciliten resolver problemáticas locales.

\section{Referencias}

André, I. y Rego, P. (2003). Redes y desarrollo local: la importancia del capital social y de la innovación. Boletín de la Asociación de Geógrafos Españoles, (36), 117-127.

Arévalo, C. (2013). Innovación e innovación social. En: Innovación social. De la teoría a la aplicación. Recuperado 25/09/2014 de: http://unilatina.edu. $\mathrm{co} / \mathrm{pdf} / \mathrm{Memorias}-\mathrm{Seminario-de-Innovacion-}$ social-Unilatina.pdf

Asian, R., Fernández, M. y Montes, O. (2013). Más allá de la RSC. Hacia un nuevo paradigma de desarrollo a través de la innovación social. Prisma social Revista de ciencias sociales, (10), 0-30.

Azuero, A. (2009). Capital Social e Inclusión Social: Algunos elementos para la política social en Colombia. Cuadernos de Administración, 25(41), 151-168.

Bacon, N., Faizullah, N., Mulgan, G. y Woodcraft, S. (2008). Transformers: How local areas innovate to address changing social needs. Nesta.

Buckland, H. y Murillo, D. (2013). Vías hacia el cambio sistémico. Ejemplos y variables para Vías hacia el cambio Ejemplos y variables para la innovación social. España. ESADE, Instituto de innovación social.

(2014). La innovación social en América

Latina.

Cano, M., Bello, E. \& Barba, E. (2012). Innovación social y capacidad de organización de las cooperativas pesqueras en el municipio de Balancán, Tabasco, México. Estudios Sociales, 20(39), 67-97.

Caravaca, I., González, G. \& Silva, R. (2003). Redes e innovación socio-institucional en sistemas productivos locales. Boletín de La A.G.E, (36), 103-116. 
Caravaca, I., González, G. \& Silva, R. (2005). Innovación, redes, recursos patrimoniales y desarrollo territorial. Revista Eure, 31(94), 5-24.

Chiriboga, M. (2003). Innovación, conocimiento y desarrollo rural. Recuperado 02/10/2014 de http://www.rimisp.org/wp-content/ u p 1 o a d s / $2014 / 01 / 0270-001896$ innovacionmanuel2.pdf

Córdoba, C., Villamarín, F. \& Bonilla, H. (2014). Innovación social: Aproximación a un marco teórico desde las disciplinas creativas del diseño y las ciencias sociales. En: Tendencias, 15(2), 3044.

De La Riva, F. (2012). Foro debate 2: participación para la inclusión y transformación social. Recuperado /07/2015 de http:/www.eapn.es/ARCHIVO/ documentos/recursos/2/Forodedebate2.pdf ISBN: 978- 846156614-3.

Durston, J. (2000). ¿Qué es el capital social comunitario? Cepal.

Fundación de la Innovación Bakinter. (2009). Innovación social: Reinventando el desarrollo sostenible. Recuperado el 22 de febrero de 2015 de: http:// www.accenture.com/SiteCollectionDocuments/ Local_Spain/PDF/Accenture_FTF_Innovacion_ social.pdf

González, C., Perpinyá, A., Tullá, A., Martín, A. y Valldeperas, N. (2014). La agricultura social en Catalunya: innovación social y dinamización agroecológica para la ocupación de personas en riesgo de exclusión. Revista de estudios sobre despoblación y desarrollo rural, (17), 65-97.

Gutiérrez, A. (2009). Innovación social: un ámbito de interés para los servicios sociales. Ekaina, (45), 151-178.

Hernández, I. \& Sánchez, O. (2014). Innovación social abierta en el diseño de una política y estrategia de formalización sostenible: un caso colombiano de gobierno colaborativo. Revista Facultad de
Ciencias Económicas: Investigación y Reflexión. 22(2), 47-61.

Jiménez, J. \& Morales, A. (2012). Social economy and the fourth sector, base and protagonist of social innovation. CIRIEC - España. Revista de Economía Pública, Social y Cooperativa, (73), 33-60

Kliksberg, B. (1997). Repensando el Estado para el desarrollo social: más allá de dogmas y convencionalismos. Reunión Mundial de Expertos.

Martín, J. (2000). Desarrollo local para un nuevo desarrollo rural. Anales de Geografía de la Universidad Complutense, (20), 85-100.

Martner, C. (1995). Innovación tecnológica y fragmentación territorial. Revista EURE-Revista de Estudios Urbano Regionales, 21(63).

Murray, R., Caulier, J. \& Mulgan, G. (2010a). The open book of social innovation. Tercer Sector, 233.

. (2010b). The Open Book of Innovation. The Young Foundation y Nesta.

Observatorio Colombiano de Ciencia y Tecnología. (2013). Memorias del Seminario Taller Los retos de la Innovación Social, DNP, Colciencias y ANSPE.

Orrego, C. \& Arboleda, O. (2006). Las organizaciones de economía solidaria: Un modelo de gestión innovador. Cuadernos de Administración, (34), 97-110.\}

Pérez, M. \& Clavijo, N. (2012). Estudios sobre innovación en la agricultura familiar. Experiencias y enfoques de procesos participativos de innovación en Agricultura. El caso de la corporación PBA en Colombia. Organización de las Naciones Unidas.

Phills, J., Deilglmeier, K. \& Miller, D. (2008). "Rediscovering Social Innovation", Stanford Social Innovation Review. 
Cindy Arcos Soto, Marlén Suárez Pineda \& Sandra Milena Zambrano Vargas

Posada, O. (2006). Manifestaciones de capital social en las cooperativas agrícolas del sector rural del departamento de Caldas (Tesis de Maestría). Universidad de Manizales, Colombia.

Rey, N. \& Tancredi, F. (2010). De la innovación social a la política pública: Historias de éxito en América Latina y el Caribe. Chile, Naciones Unidas.

Reyes, L. (2011). La innovación social como atributo de la actividad informacional. (Spanish). Ciencias de la información, 42(2), 5-10.

Unesco (2015). Educación, Poblaciones rurales. Recuperado 25/07/2015 de http://www.unesco. org/new/es/education/themes/strengtheningeducation-systems/inclusive-education/ruralpeople/

Vera, E. \& Lavalle, A.G. (2010). La innovación democrática en América Latina: tramas y nudos de la representación, la participación y el control social. Centro de Investigaciones y Estudios Superiores en Antropología Social (CIESAS).

Westley, F. (2009). "Making a Difference: Strategies for Scaling Social Innovation for Greater Impact", Innovation Journal, Vol. 15, No 2 . 